\title{
Don't stress aggressors
}

\author{
THE BIOLOGY \\ OF VIOLENCE

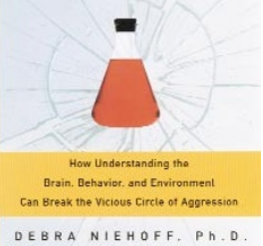 \\ the Vicious Cycle of Aggression \\ by Debra Niehoff \\ The Free Press, New York, 1998. \$25 \\ hardcover, pp 353 \\ ISBN 0-684-83132-5 \\ Reviewed by James Blair
}

The Biology of Violence: How Understanding the Brain, Behavior, and Environment Can Break

Given the rather slow first two chapters and somewhat dull cover visuals, readers might be forgiven for not persisting with Debra Niehoff's book, The Biology of Violence. This would be their loss, however, for it is easily the most entertaining general text I have read since Joe LeDoux's The Emotional Brain. The two books partially overlap in being primarily concerned with the brain systems that respond to threat. However, where LeDoux concentrated on the role of the amygdala in emotion, Niehoff discusses the role of the hypothalamus, amygdala and periaqueductal gray area in the initiation of aggression as a response to threat.

There is a rather striking picture in a paper by Caroline and Robert Blanchard depicting the responses of a rat to an approaching human. First the rat freezes. Then, as the human draws closer, it attempts to escape. Finally, if escape is prevented and the human draws closer still, the rat attacks. Extreme activation of the animal's response system for threat causes the initiation of a defensive attack. The central thrust of Niehoff's book is that aggressive humans are individuals whose response system for threat is primed, through environmental stressors, to be almost at the attack level. Any mild stressor may make them initiate a defensive attack. This is an attractive idea, not least because it gives some credence to the idea of a biology of violence. Not too long ago the Ciba Foundation published The Genetics of Criminal and Antisocial Behaviour. Although the book was interesting, the title was unfortunate. Simplistically, it is inconceivable that there are genes for specific criminal behaviors. However, there may be, as indeed Niehoff argues, genes that modulate the sensitivity of the system that

James Blair is at the Institute of Cognitive Neuroscience, University College London, Alexandra House, 17 Queen Square,

London WC1H $3 A R, U K$.

e-mail: j.blair@ucl.ac.uk responds to threat. It is possible that certain genes might result in the development of a threat system that has a lower threshold for activation, and thus an individual who initiates violence at a lower level of threat.

Niehoff suggests that there is a 'vicious cycle' such that early environmental threats, such as abusive parents, cause neurobiological changes that increase sensitivity to threat and thus, according to her argument, increase the probability of aggression. The individual's aggression leads to more environmental abuse and the perpetuation of the cycle. Through this central focus, Niehoff's book provides a very good guide to the neural architecture involved in the response to threat, its neurochemistry, hormonal influences on stress and even genetic influences on threat and aggression. The chapters on the neurochemistry of aggression and hormonal influences are particularly well thought out. The basic arguments are presented with a tight focus, and she deals appropriately with simplistic arguments, for example that aggression results from too much testosterone. Indeed, the writing is clear, entertaining and full of colorful metaphors. Whenever I think of the cerebellum in the future, I am sure that I will remember it "cascading over the sides of the brain stem like a fleshy garden". The analogy of the endocrine system to the Pony Express system of the American West was particularly delightful.

My main criticism of the book is its relentless concentration on aggression as a threat response. It is difficult to be sure how much interhuman violence really is of this form, and Niehoff does not provide any specific data on this issue, probably because none is available. Kenneth Dodge has found that aggressive individuals are more likely to show 'hostile attribution biases'; they are more likely to judge, for example, that an individual knocking into another individual did so out of hostile intent rather than by accident. However, these biases explain only a small degree of the variance in aggression. In addition, the Blanchards and others have shown that aggression certainly is a response to threat in most animal species, so Niehoff's argument is plausible. Yet it is unclear how applicable the thesis is. How can we determine whether the man who killed his stepchild did so because the aversive stimulus of the child's crying pushed his threat system over the edge?

Indeed, Niehoff does not make the mistake of considering all aggression to be a response to threat. She clearly lays out other causes of high aggression levels, including describing psychopathy as another, but completely different, 'stress response disorder'. However, her discussion of other causes of aggression is much less satisfactory than her discussion of defensive aggression. For example, the discussion of psychopathic individuals is very brief indeed. Robert Hare has identified a psychopathy syndrome that is made up of two distinct components: a interpersonal-affective component marked by a lack of guilt and empathy and an antisocial behavior component marked by high levels of aggression and crime that appears early in life. This definition has the significant advantage for research that it identifies a relatively homogeneous population, unlike the DSM diagnosis of antisocial personality disorder (with which Niehoff inaccurately equates it). Her brief coverage of psychopathy is unfortunate, as it is clear that psychopathic criminals are far more likely to be aggressive than criminal individuals without psychopathy. At the anatomical level, there is minimal reference to orbitofrontal cortex, despite the very clear data provided by researchers like Grafman on Vietnam War veterans. Acquired lesions to orbitofrontal cortex, and not frontal cortex more generally or more posterior regions, are risk factors for violence.

Even as I make the above criticisms, though, I feel slightly pedantic. The book reviews a considerable literature interestingly and well, grouping it around a central theory that is at least provocative, even if its applicability is unclear. A reasoned and intelligently argued position on the biology of violence is rarely read, and the idea of aggression as a threat response allows a host of testable predictions. For example, aggressive individuals with heightened stress responses should show similar task performance to patients with post-traumatic stress disorder, that is, augmented startle reflexes to loud noises following visual threat primes and heightened sensitivity to threatening words. Certainly, next time I find myself in a hostile situation, I will be careful not to stress my potential aggressor. 